\title{
LECTURE
}

\section{Pulmonary Diffusing Capacity: Problems in Its Definition and Determination}

\author{
Johannes Piiper \\ Department of Physiology, Max Planck Institute for \\ Experimental Medicine, Göttingen, FRG
}

(Received for publication on April 18, 1986)

\begin{abstract}
Pulmonary diffusing capacity or diffusive gas/blood transfer conductance of the lungs, DL, defined as transfer rate divided by effective partial pressure difference, is a quantitative parameter for the fundamental property of lungs to allow gas exchange by diffusion. Its accurate definition and determination are complicated by a number of difficulties arising from several sources which may be grouped as follows: (1) functional inhomogeneity of parallel lung units (ventilation/diffusion/perfusion inequality); (2) heterogeneity of diffusion barrier (alveolar space; tissue layers separating blood from gas; plasma; red blood cell); (3) limitation of red cell/plasma equilibration by processes other than gas diffusion (chemical reaction; ion exchange); (4) anomalous blood/gas equilibria (for $\mathrm{CO}_{2}$ ).
\end{abstract}

Key words: diffusion, functional inhomogeneity, equilibration, gas transfer

\section{Introduction}

We owe the concept and the first determination of pulmonary diffusing capacity (DL) to Marie and August Krogh (1909) who introduced (what we now call) the carbon monoxide (CO) single-breath or breath-holding method. After World War II the methods for measurement of $\mathrm{Dr}$ for $\mathrm{CO}$ and for $\mathrm{O}_{2}$ were elaborated and introduced into clinical laboratories were the single-breath Duoo has become a basic and important index for evaluation of the pulmonary gas exchange function.

Reprint requests to: Dr. Johannes Piiper, Department of Physiology, Max Planck Institute for Experimental Medicine, D-3400 Göttingen, FRG 
In this lecture, the physiological background of the concept of the pulmonary diffusing capacity and the problems of its determination will be analyzed on the basis of more recent research in the field of alveolar gas exchange. The main complications arising from the fact that lung structure and function deviate from the simple basic model, uniform alveolar space separated by a diffusion barrier from pulmonary capillary blood, will be summarized.

A more detailed and extensive treatment, with references, is presented in: J. Piiper and P. Scheid, Blood-gas equilibration in lungs, in Pulmonary Gas Exchange, Vol. I, pp. 131-171, ed. by J. B. West, Academic Press, New York, 1980.

\section{(1) Functional inhomogeneity of parallel lung units}

It is well known that unequal distribution of alveolar ventilation to pulmonary blood flow gives rise to inefficient gas exchange which may be quantified by an alveolararterial $\mathrm{Po}_{2}$ difference. When this inhomogeneity is not taken into account, Duos as conventionally calculated from alveolar-arterial $\mathrm{Po}_{2}$ difference in hypoxia will be underestimated. The use of the so-called ideal alveolar gas reduces the error, but in most cases does not eliminate it. Similar, but lesser, effects will occur with determination of steady state Duoo, and with single breath (breath-holding) methods for which the distribution of ventilation to alveolar volume is of importance.

Less known, however, is the effect of unequal distribution of De to blood flow (or to ventilation or to lung volume). This may be due unequal distribution of vascular resistance, of capillary length, or to local thickening of the gas-blood diffusion pathway; its extreme case is a complete shunt. It may well be that an important part of the increase of $\mathrm{DLO}_{2}$ in hypoxia as compared to normoxia is due to a reduction of the effects of $\mathrm{Du} /$ blood flow inhomogeneity on gas exchange efficiency.

Another new aspect of unequal distribution is the unequal distribution of airway resistance to diffusion as exhibited by the asymmetric lung model of Paiva and Engel. The overall effect on $D_{L}$ of all these inhomogeneities is similar: underestimation if not properly taken into account.

\section{(2) Heterogeneity of the diffusion barrier}

In the ideal model for diffusion the diffusion barrier is a homogeneous membrane separating alveolar gas from blood; there is resistance to diffusion neither in the alveolar gas nor within the blood. In reality, these assumptions are not valid.

(a) Diffusion resistance in alveolar space (stratification). The role of stratified inhomogeneity due to incomplete mixing of inspired gas with resident lung gas in limiting pulmonary gas exchange is controversial, and few are the attempts to quantify it in terms of a "stratificational diffusing capacity." If present, it would affect gas exchange 
in a manner similar to alveolar dead space due to ventilation of unperfused lung regions. It may well be that part of the alveolar dead space is due to incomplete mixing of alveolar gas.

(b) Diffusion resistance of plasma. The effective diffusion resistence of plasma in pulmonary capillaries is difficult to estimate because of lack of knowledge on the streaming dynamics, but it may be of considerable importance.

(c) Diffusion across red cell membrane and within red cells. In spite of considerable theoretical and experimental effort, it cannot be stated with any certainty whether the main resistance to $\mathrm{O}_{2}$ uptake resides in the tissue barrier or within the red blood cells. However, there is general agreement that the RBC membrane has a negligible limiting effect.

(d) Chemical reactions and ion exchange as limiting factors. For alveolar $\mathrm{O}_{2}$ exchange there exists no evidence for chemical reaction kinetics of $\mathrm{O}_{2}$ with hemoglobin to exert limiting influence on alveolar-arterial equilibration of $\mathrm{O}_{2}$. Also results of red cell/medium equilibration with rapid reaction stopped-flow techniques yield no evidence for reaction limitation in the hypoxic range, but leave this possibility open for the normoxic range. Also for $\mathrm{CO}$ there seems to be little reaction limitation in hypoxia when free hemoglobin is present, but a definite reaction limitation is seen at normoxic and hyperoxic oxygen levels.

\section{(3) Limitation of red cell/plasma equilibration of processes other than gas diffusion}

From Krogh diffusion constants for water or tissue, a $\mathrm{Duco}_{2} / \mathrm{DLO}_{2}$ ratio of about 20-25 is expected. The much smaller experimental ratio, about 5 , indicates that there must exist some addition resistances to $\mathrm{CO}_{2}$ transfer. This effective equilibration resistance to $\mathrm{CO}_{2}$ transfer. This effective equilibration resistance is attributed to the limiting role of the "secondary" processes accompanying $\mathrm{CO}_{2}$ transfer: dehydration of bicarbonate (catalyzed by carbonic anhydrase, but possibly not sufficiently), $\mathrm{HCO}_{3}{ }^{-} / \mathrm{Cl}^{-}$exchange through the red cell membrane, slow equilibration of $\mathrm{H}^{+}$across the red cell membrane. It appears that alveolar-capillary equilibration of $\mathrm{CO}_{2}$ may be slower than that of $\mathrm{O}_{2}$ (in normoxia), and $\mathrm{CO}_{2}$ exchange may retard $\mathrm{O}_{2}$ equilibration through the Bohr-Haldane effect.

\section{(4) Anomalous blood/gas equilibrium}

During the last two decennia a number of experimental studies on man and animals have indicated that in equilibrium $\mathrm{PcO}_{2}$ in blood in equilibrium with alveolar gas is measurably (up to by about 10-20 Torr) lower than alveolar gas $\mathrm{Pco}_{2}$. However, in repeat studies performed by other investigators, this remarkable finding was not reproduced. The controversy has not yet been solved. If this finding was correct, the efficien- 
cy of lungs in eliminating $\mathrm{CO}_{2}$ should be (much) higher than classically assumed for "ideal" lungs. Also, the applicability of the concept of De should be critically examined and modified. No analogous finding has been reported for alveolar/capillary $\mathrm{O}_{2}$ transfer.

\section{Conclusion}

The various problems mentioned in this lecture render the accurate determination of pulmonary diffusing capacity difficult, particularly in patients with pulmonary disease. The knowledge of these problems is important for making appropriate corrections whenever possible. 